Vol.02/ No. 02

Pages: 77-99

http://irojournals.com/aicn/

DOI: https://doi.org/10.36548/jaicn.2020.2.002

\title{
Applying a 6-axis Mechanical Arm Combine with Computer Vision to the Research of Object Recognition in Plane Inspection
}

Joy Iong-Zong Chen,

Department of Electrical Engineering,

Da-Yeh University,

No. 168 University Rd., Dacun,

Changhua 51591, Taiwan

E-mail: jchen@mail.dyu.edu.tw,

Jen-Ting Chang

Department of Electrical Engineering,

Da-Yeh University,

No. 168 University Rd., Dacun,

Changhua 51591, Taiwan

\begin{abstract}
The study of a robotic arm copied with 3D-printer combines computer vision system with tracking algorithm is proposed in the paper. Moreover, the designing to the intelligent vehicle system with the integration of electromechanical for planning to apply it to the operations in various fields is presented too. The main purpose of this work tries to avoid the complicated process with traditional manual adjustment or teaching. It is expected to achieve the purpose that the robotic arm can grab the target automatically, classify the target and place it in the specified area, and even accurately realize the classification through training to distinguish the characteristics of the target. Eventually, the mechanical arm's movement behavior is able to be corrected through a real-time image data feedback control system. In words, with the experiment that the computer vision system is used to assist the robotic arm to detect the color and position of the target. By adding color features for algorithm training as well as through human-machine collaboration, which approves that the proposed algorithm has well known that the accuracy of target tracking definitely depends on both of two parameters include "object locations" and the "illustration direction" of light source. The difference will far from $75.2 \%$ to $89.0 \%$.
\end{abstract}

Key words: Robotic arm, Computer vision system, Tracking algorithm, Real-time object detection

ISSN: 2582-2012 (online)

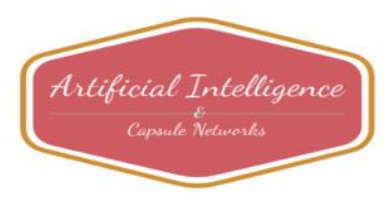


Journal of Artificial Intelligence and Capsule Networks (2020)

Vol.02/ No. 02

Pages: 77-99

http://irojournals.com/aicn/

DOI: https://doi.org/10.36548/jaicn.2020.2.002

\section{Introduction}

Both the purpose of optimizing today's industrial production and integrating various technologies is the goal of Industry 4.0. Thus, it is critically important issue to integrate the information system with the information in the real space through the Internet, such as, the IoT (Internet of Things), big data, AI (artificial intelligence) and other diverse customized manufacturing. A single-function target is adopted in traditional industrial manufacturing with most of the mechanical arms. The advantage of combine with integrated system of complete work is that they can complete quick and accurately to pick an objects and to move to a suitable place at the same time. Such behavior can reduce the time for complicating the operations convenience, and also totally improve the allocation of human resources [1]. However, under the influence of globalization in order to apply those substantial monitoring records from the process of production, to make more advanced technical planning with the assistance of high technique is necessary. The consideration to apply an auxiliary tool that can bring huge profits to the enterprise, apply precise robotic arms maximizes the usage of all machine equipments in the factory, and even to monitor the signal for handling the production of components in the factory are some most strategies carried out in a factor. Especially, if an enterprise is trying to transform the flow of manufacturing. Record and report timely to information are importantly required, such as storage, storage location, management personnel, etc., and effectively predict the loss of the machine, fault maintenance, and even reduce operating costs caused by misjudgment. Moreover, how to embed human thinking and behavior capabilities into a robots let it becomes smarter, more agile, and even low-cost, which is worth thinking. It is possible to establish a new model of cooperation between the human and machine. Under the cooperation of man and machine, robots can not only help humans complete cumbersome and dangerous special tasks, but also transmit the lowest-level information. Therefore, by using of back-end information processing with the excellent precision and flexibility of human beings, such fast and complete systematic approach can

ISSN: 2582-2012 (online)

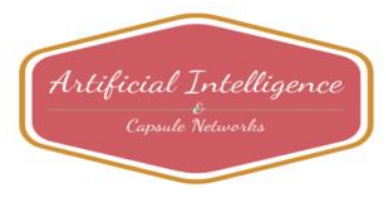


Journal of Artificial Intelligence and Capsule Networks (2020)

Vol.02/ No. 02

Pages: 77-99

http://irojournals.com/aicn/

DOI: https://doi.org/10.36548/jaicn.2020.2.002

upgrade production methods and continue to remain competitive. Based on the discussed issues previously, in this study is focusing in applying a robot arm automatically to perform the grasping and classification operations, and then uses computer vision software to detect the position and color characteristics of the target object for recognition [2].

Recently, there are many studies in the case of manufacturing promotion for automobile industry, and some large industrial manipulators were often seen, as well as parallel manipulators that were fast to pick and place on the production line. In words, their common ideas are attending to control an arm machine operates repeated on a command comes from human being. For example, clamp a fixed object within the preset range. Besides, in traditional industrial control, most of them is adopting PLC (programmable logic controllers) for command control, for instance, the technique of PLC could be implied in the operation process for a robotic arm. On the other hand, the application to apply PLC could take a lot of modification time for adjusting the new operating procedures. Thereafter, the control logic of arm can be approached by editing the program instructions and continue to work according to the set mode, moving, picking, rotating and unloading, these previously mentioned actions are fixed easily. Hereafter, to explore those studies in the case of manufacturing with advanced technologies are as follows. The development of robotic arm for 3 degree of freedom directing by using inverse kinematics is proposed in [3] for application of industrial automation with PLC. This paper describes the requirement of reliability, accuracy, flexibility and security required for threading and grinding applications. In [4] an arm embedded into a complex CNC cutting system with multiple motion axes and cutting heads is presented, which has the main challenges, problems, and steps involved in software implementation of robotic arm control. The control algorithm is implemented into existing complex software of real-time control system. Apart from, by using servo actuator for the action of gripper is designed for force calculation is illustrated in [5-6]. This embedded sensor will be integrated with the controller for force control while grasping. The grasping process leads faster ISSN: 2582-2012 (online) 
Journal of Artificial Intelligence and Capsule Networks (2020)

Vol.02/ No. 02

Pages: 77-99

http://irojournals.com/aicn/

DOI: https://doi.org/10.36548/jaicn.2020.2.002

compared to earlier designed grippers.

In summer, in this study the developed open source computer vision software combined with Open-CV package library for image processing are investigated. Then, a web camera is selected for the visual sensor with the general USB interface network. In the paper, the object tracking algorithm called as Camshift is used to track the target, and the learning model including the mean shift is applied to the cluster data (Cluster analysis). Specifically, the training data set is collected from the color code data of the target's color characteristics. Then, the experimental design selects the servo motor as the joint control of the mechanical arm, and the drive circuit is deployed to connect the controller. The organization of the paper is combined with five sections described as below, after the Introduction section the innovative mechanism of object tracking for the traditional ways of factor producing is expressed in Section II. In Section III is to discuss with the machine training with collected data set and algorithms for the smart robot. The deployment and the results from the experiments are presented in Section IV. Eventually, there is a short conclusion draw in Section V.

\section{The Algorithm of the Object Tracking}

The algorithm for preparation of object tracking and plane inspection is described in this section detail. In order to look for the algorithm suitable for real-time tracking, it depends on many conditions includes easy to implement, the amount of calculation is small, if the target moves too fast, the volume size, and whether it is blocked. The target tracking effect will be lost if the advantages and those conditions can't be deployed completely.

It is known that one of the famous algorithm for applying to track and identify the color features of the target is called as Camshift algorithm [7]. By using the deployment of Camshift algorithm for object tracking is adopted in this article. The hue value and area of the

ISSN: 2582-2012 (online) 
Journal of Artificial Intelligence and Capsule Networks (2020)

Vol.02/ No. 02

Pages: 77-99

http://irojournals.com/aicn/

DOI: https://doi.org/10.36548/jaicn.2020.2.002

target will be judged through the features of the object, when it is going into the range as the target. Therefore, the color and shape of the target become the input conditions of the object for tracking algorithm. Eventually, the most similar features are going to track in the screen, once the target that matches to the predicted one. Certainly, the first step to define the input conditions for tracking the target is necessary. Next, follows to select the area on the screen and calculate the color histogram of the possible candidate target finally. Herein, the back projection calculation is going to figure out the color histogram of the target object which is scanned in the input screen. Then the result of the back projection calculation is selected according to the return tracking area of the target object, and which passed to the Camshift algorithm for obtaining the real object what is the prediction. On the other hand, the color information of the target's color histogram is converted into a color probability density distribution, and by backing projection calculation with the means of map (reverse projection) to obtain the target's screen position. At the first time, in order to obtain the current center position of the target, the size and position of the tracking screen for initial initialization of the target are adjusted appropriately. In summary, the operation steps for the specifically process of CAMSHIFT object tracking algorithm is shown in Fig. 1, and the stages are described as follows.

ISSN: 2582-2012 (online) 
Journal of Artificial Intelligence and Capsule Networks (2020)

Vol.02/ No. 02

Pages: 77-99

http://irojournals.com/aicn/

DOI: https://doi.org/10.36548/jaicn.2020.2.002

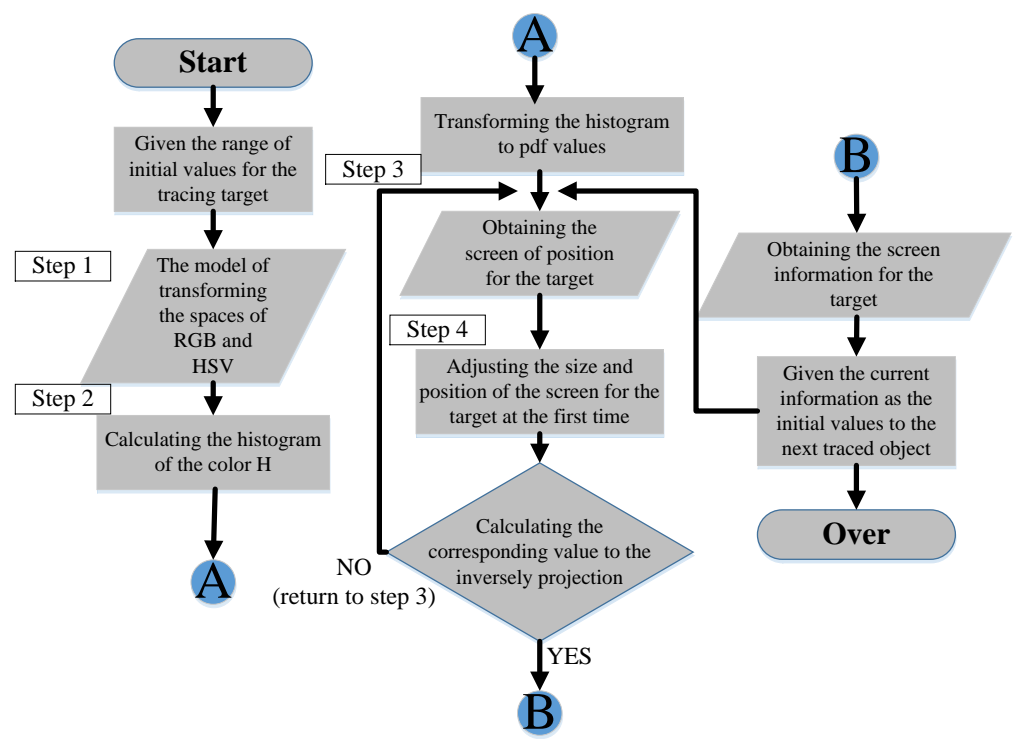

Fig. 1. The operation steps for CAMSHIFT algorithm

The operation of CAMSHIFT algorithm is completed with 4 steps running. First of all is as the step 1 shown in Fig. 1, which is the action for transforming color space. It is well known that, the RGB color space generally is more sensitive to large variate in ambient light sources. Hence, one of the skills is converting the RGB color space into an HSV (Hue, Saturation, Lightness) model to avoid the effect of lighting conditions on tracking an object. Then turn to the step 2 where the hue value $\mathrm{H}$ in the HSV model is calculated as a color histogram, and the color distribution or the number of pixels are represented graphically. The probability of the color appearing in the image is following replaced to get the distribution map. The got results contain almost the relevant information of the target, for instance, the red area can be obtained as the relevant information for an apple histogram. The back projection calculation becomes the $3^{\text {rd }}$ step indicated in Fig. 1. Each pixel comes in the input image is corresponding to the color histogram of the target, and the probability of the target pixel within this detection range will be figured out. Normally, the probability of pixels outside the detection range is noted as " 0 ". This process is the aforementioned back projection method and the probability distribution map is called as gray-scaled. Iteratively, the implement of backward projection is

ISSN: 2582-2012 (online) 
Journal of Artificial Intelligence and Capsule Networks (2020)

Vol.02/ No. 02

Pages: 77-99

http://irojournals.com/aicn/

DOI: https://doi.org/10.36548/jaicn.2020.2.002

applied to search the target corresponding value. The size and position of the next target will be calculated and adjusted according to the corresponding value shown in the tracking screen and as the new initial value.

In fact, the implementation of this algorithm is able to be found in the OpenCV ${ }^{\mathrm{R}}$ computer vision software library. Their scenarios are shown in Fig. 1 and Fig. 2, respectively.

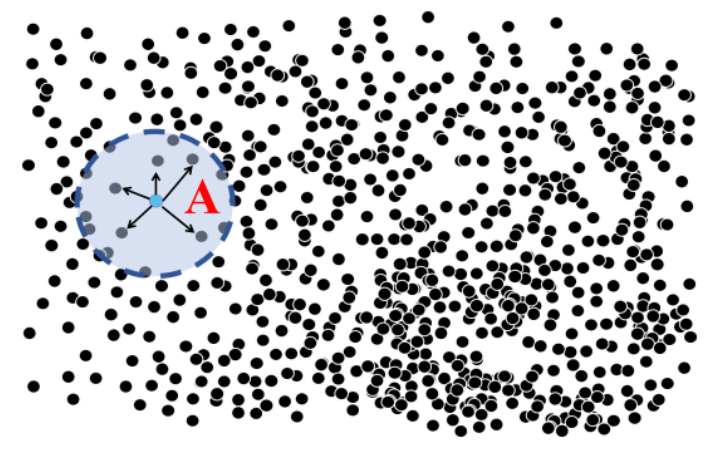

(A)

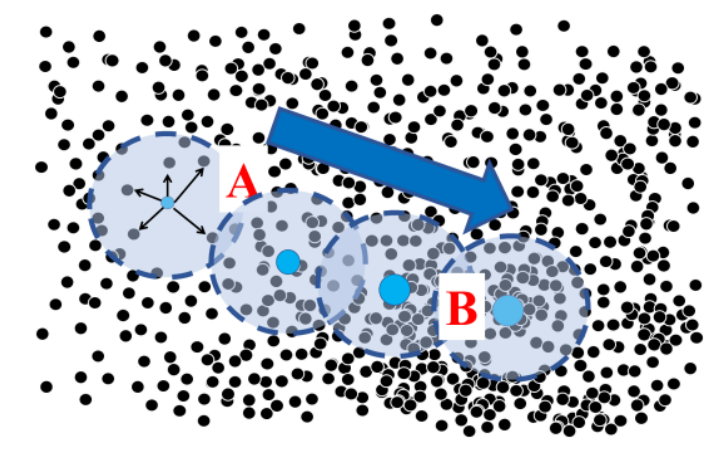

(B)

Fig. 2. (A) A center point position for calculating the vector average

Fig. 2. (B) A center point position movement for calculating the mean drift

Specifically, the establishment to the model of data set aggregation is an important issue for addressing. Thus, herewith many stages to arrive at the purpose of the target tracking are claimed as follows.

1). Calculate the average offset while the position of the initial center point is obtained. As point A shown in Fig. 2, selecting a center point position calculate the vector average of the distance from the point contained in the area where the center point is located to the center point. The new results by calculating the offset average value of this vector average are

ISSN: 2582-2012 (online)

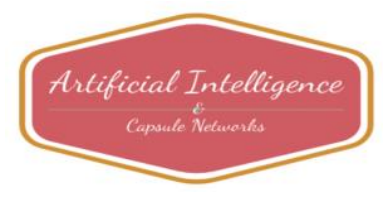


Journal of Artificial Intelligence and Capsule Networks (2020)

Vol.02/ No. 02

Pages: 77-99

http://irojournals.com/aicn/

DOI: https://doi.org/10.36548/jaicn.2020.2.002

obtained by assuming that, $I=f(x, y)$. The last function is the color probability map evaluated by back projection form the histogram of the HSV color space.

If $W$ expresses the initial position and size of the mobile window. The pixels $(x, y)$ contained in the calculation window is given as

$$
\begin{aligned}
& M_{00}=\sum_{x, y} I(x, y) \\
& M_{10}=\sum_{x, y} x I(x, y) \\
& M_{01}=\sum_{x, y} y I(x, y) \\
& \Rightarrow x_{c}=\frac{M_{10}}{M_{00}}, y_{c}=\frac{M_{01}}{M_{00}}
\end{aligned}
$$

where the core of $\mathrm{W}$ is presented as $\left(x_{c}, y_{c}\right)$. The last equation is satisfied as long as the amount of movement of $W$ exceeds a certain range value and the maximum number of iterations is not reached.

2 ). To figure out the new center point position, and move the center repeatedly to reach the area with the highest density. The results will lead to move the center point A to the area where the average value of the offset is obtained. Repeat the moving direction all the time to obtain the best solution for the center point position, as point B shown in Fig. 3. From the above process, it is clear that the mean drift model could be repeatedly used to carry out the average distance.

ISSN: 2582-2012 (online) 
Journal of Artificial Intelligence and Capsule Networks (2020)

Vol.02/ No. 02

Pages: 77-99

http://irojournals.com/aicn/

DOI: https://doi.org/10.36548/jaicn.2020.2.002

Hereafter, there is an example explained for the calculation of the Camshift algorithm. The parameter of the ellipse estimated from the second moment of the probability distribution $I=$ Pskin skin color. The next flow of Camshift algorithm will expand the window $W$ and calculate the $99 \%$ ellipse for all included points, as the average offset reaches convergence. In fact, these contained points belong to a 2D Gaussian distribution, the parameters of the ellipse can be predicted from the second moment of the area $W$. Considering the central moments as order $i$ of $\mathrm{x}$ and order $\mathrm{j}$ of $\mathrm{y}$ are defined as,

$$
\mu_{i, j}=\sum_{x, y}\left(x-x_{c}\right)^{i}\left(y-y_{c}\right)^{j} I(x, y)_{(2)}
$$

where last equation expresses that the summation is performed on the enlarged window size $W$, let $\theta$ denoted as the direction of the ellipse and given by

$$
\theta=\arctan 2\left(2 \mu_{11}, \mu_{20}-\mu_{02}+\sqrt{4 \mu_{11}^{2}+\left(\mu_{20}-\mu_{02}\right)^{2}}\right)
$$

where the function arctan 2 is the operant inverse tangent of a four quadrants. The main moment of inertia of the ellipse can be evaluated as

$$
\begin{aligned}
& I_{\mathrm{m} \mathrm{a}} \overline{\overline{\mathrm{x}}} \sum_{x, y}\left(( x - x _ { c } I ) x \left(y, \theta \leftrightarrow \boldsymbol{g} \& y_{c} I\left(\begin{array}{lll}
x y & ) \theta^{2}
\end{array}\right.\right.\right.
\end{aligned}
$$

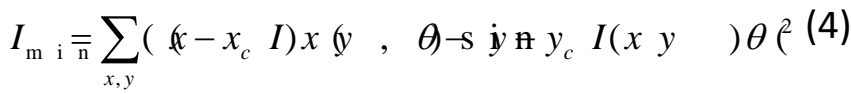

, and which can be simplified as

$$
\begin{aligned}
& I_{\text {max }}=\mu_{20} \cos ^{2} \theta+2 \mu_{11} \cos \theta \sin \theta+\mu_{02} \sin ^{2} \theta \\
& I_{\text {mix }}=\mu_{20} \sin ^{2} \theta+2 \mu_{11} \cos \theta \sin \theta+\mu_{02} \cos ^{2} \theta
\end{aligned}
$$

ISSN: 2582-2012 (online) 
Journal of Artificial Intelligence and Capsule Networks (2020)

Vol.02/ No. 02

Pages: 77-99

http://irojournals.com/aicn/

DOI: https://doi.org/10.36548/jaicn.2020.2.002

where $a$ is the dimensions of the long axis and $b$ is short axis of the ellipse. The ellipse is at an angle greater than $45^{\circ}$ compared to the horizontal if the condition is a $<\mathrm{b}$. It is easily to understand that the lengths are interchanging at $\theta=\pi / 2-\theta$. By the back projection of the vertices of the ellipse on $\mathrm{x}$ and $\mathrm{y}$ axes from the next image, the height and width of the initial window of the mean shift can be derived as

$w=\max (a \cos \theta, b \sin \theta), h=\max (a \sin \theta, b \cos \theta)(7)$

In words, the size of the assigned window changes to the movement of the center point position simultaneously until it converges. In each iteration, there is a given size window will apply to the mean shift. The process is repeated with a new window centered on the position found by the mean shift, after the mean shift has converged. However, its size depends on the zeroth moment of the spatial distribution of the previously calculated skin color probability and movement distance averagely [7-8].

\section{Description to the Data Set and Training}

The successful possibility for the mentioned tracking algorithm in this article depends on an important key factor which is the judgment of color feature. It is necessary to include both the definition what color is required or even the shade of the color, and how to convert the RGB primary colors into the HSV color model. The collection of color data is a very important preprocess in the case of solving these problems. Furthermore, in the beginning try the best possible way to enquire much more color code tables under the free license of open data on the Internet. Generally, the tables contain several conversion methods, and the RGB (red, green and blue) color models have the main usage in electronic system image display. For example, the three primary colors are background for TV display. The CYMK color

ISSN: 2582-2012 (online) 
Journal of Artificial Intelligence and Capsule Networks (2020)

Vol.02/ No. 02

Pages: 77-99

http://irojournals.com/aicn/

DOI: https://doi.org/10.36548/jaicn.2020.2.002

combination mode is adopted in color printing, that is, the colors of Cyan, Magenta, Yellow, and black are able to be adjusted and mixed into new colors. Besides, the HSV color model is mainly implied in application of computer vision. A color is selected for specific occasions according to the application requirements. $\mathrm{H}$ is the hue value, $\mathrm{S}$ is the saturation, and $\mathrm{V}$ is the brightness. Finally, there is a else method expressed in hexadecimal code, such as, the codes of \# 000000 and \#FFFFFF present black and white, respectively. All over the mentioned above is the basic investigation and understanding about the discussion to color.

\subsection{The Application of Computer Vision}

The study to the field of industrial automation the computer vision is one of the important application technologies with the analysis to the processing image information.

Consider several of measured conditions which are important factors affecting computer vision interpretation. The most directly relevant to the effect of the implement functions of positioning and classification is involving the external light source. Usually, the illuminate in the correct way to highlight the characteristics of the observed object, such as, color, shape, size, which will ensure that the lens can successfully acquire the image for processing. Accordingly, the changes of the ambient light source will generate instability quality of the image data. Hence, recovery or adjust to the light source by using of appropriate protective measures can create the opportunity to solve the measurement error. Correspondingly, in the report the test environment of the inspection functions is designed as a structure built with general webcam lenses and an embedded system assigned as Raspberry $\mathrm{Pi}^{\circledR}$. On the other hand, the installed OS (operating system) of the embedded system is Linux, and it has the appearance similar to the size of a business card. There is the input and output interfaces called as GPIO (General purpose I/O) integrated into a desktop computer for The Raspberry $\mathrm{Pi}^{\circledR}$. The skeleton of GPIO has 40 pins that can be expanded and compiled, and it provides an

ISSN: 2582-2012 (online)

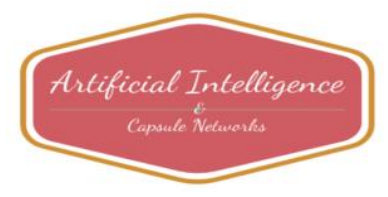


Journal of Artificial Intelligence and Capsule Networks (2020)

Vol.02/ No. 02

Pages: 77-99

http://irojournals.com/aicn/

DOI: https://doi.org/10.36548/jaicn.2020.2.002

excellent environment for developing applications. Moreover, there are lots of OpenCV development libraries with open sources types for image processing the embedded system. Normally, it is utilized to program the codes quickly, providing many programming language interfaces, and even suitable for the machine learning algorithm applications.

\subsection{The Feature Data Training and Target Positioning}

The procedure of training to the feature of a selected object is explained in the principle of color operation method for an image processing. As mentioned in the 3.3 training data set, the color code table data is collected, and the HSV color model is used for detection. The color seen by the human eye comes from the light source illuminating the surface of the object and imaging it into the brain through reflection. The color space explained in terms of color characteristics was proposed by A. R. Smith in 1978. In addition to the general RGB color spaces, the HSV model represents the uniqueness of a color. RGB represents the three primary colors R-red, G-green, and B-blue, respectively. In image processing, vectors are usually used to represent color values, such as white $(255,255,255)$, and black $(0,0,0)$. These numbers are quantized into 255 numbers, and there are $256 * 256 * 256$ colors in three-dimensional space. Besides, three parameters are represented as the HSV model, hue $(\mathrm{H})$, saturation (S), and brightness $(\mathrm{V})$. Herewith, 'Hue' is the attribute of color, such as white, black, etc. are the name of the color. 'Saturation' is the purity of the color which has the feature of the higher the saturation, the more purity of color. In addition, the lower the gray and the brightness is the brightness of the color. The 'brightness' value is related to the brightness of the light source, and generally the value is arranged from $0 \%$ (black) to $100 \%$ (white). The HSV model is adopted as computer machine vision and selects a color in a

ISSN: 2582-2012 (online) 
Journal of Artificial Intelligence and Capsule Networks (2020)

Vol.02/ No. 02

Pages: 77-99

http://irojournals.com/aicn/

DOI: https://doi.org/10.36548/jaicn.2020.2.002

specific application environment.

It has the beneficial to enhance the accuracy for image recognition after the removal of bright colors from the original image. Filtering noise and leave necessary information are the main purpose of the removal activity. Generally, the Gaussian blur theory is applied both to smooth the image and blur the image. A processing method of the algorithm is used to regenerate the pixel value of the map. Normally, the Gaussian function is selected as the way to generate the corresponding result of the normal distribution. The matrix formed by the pixels can be obtained by convolution filtering the target pixels and to complete the conversion.

'Dilation' and 'erosion', are two important operations in the morphology of image processing. It is known that there are three main purposes, the first is to find the regions of the maximum and minimum of the image, segment the independent image elements, and then to obtain the image gradient. The dilation operation is try to search the local maximum value and it is expanded in the highlighted part. Thus the original image brightness is increased to highlight the brightest area. The corrosion is an application of reverse operation. The dark area of the image is going to appear after finding the minimum value, and the retain the contour edge of the object is completed by looking for the difference between the inspection of expansion and corrosion. Anyway, the function library provided by the open source computer vision OpenCV is used for development in the stage of actual test, and the general color photo should be converted into the steps of expansion and corrosion.

Scanning the binary image can leave the main distinguishing object features after the step of grayscale is finished. This operation is useful for promoting the ability of image recognition. Based on the determination of color by using color code data for feature training,

ISSN: 2582-2012 (online) 
Journal of Artificial Intelligence and Capsule Networks (2020)

Vol.02/ No. 02

Pages: 77-99

http://irojournals.com/aicn/

DOI: https://doi.org/10.36548/jaicn.2020.2.002

and selecting the blue system as the main detection target of the machine vision system proposed the article. The specified process flow chart is shown in Fig. 8.

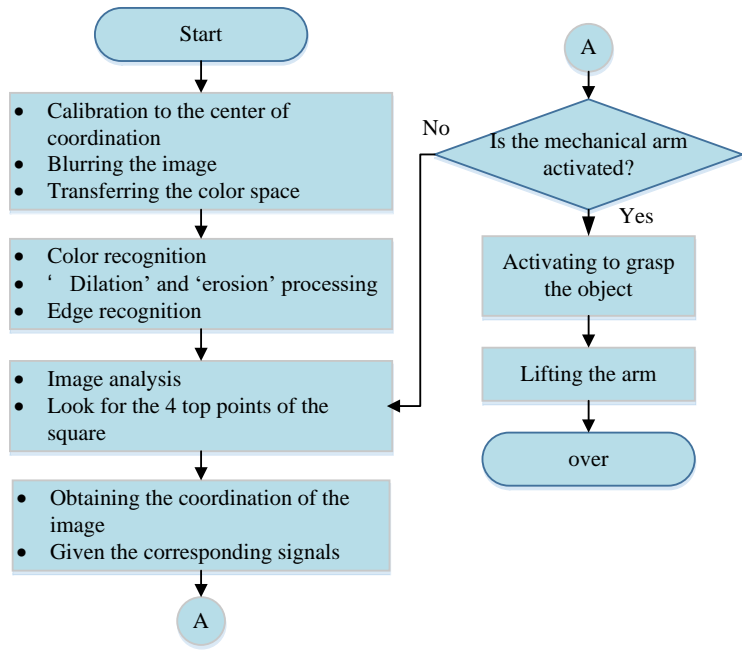

Fig. 8 The flow chart for the detection target of the machine vision system

The image processing applications also have the function to identify the shape of objects except the feature recognition. The judgment of shape can be done by setting the appearance of the target. Certainly, it is able to be designed by suitable gripping mechanism according to the shape of the target. The hardware composition including combines the mechanical arm, the camera, and mainly uses the lens to obtain the position information of the target object, and draws the edge and the outline. In the paper, the calculation of the spatial position as well as adjust the visual calculation method according to the application are deployed. It can accurately capture the target in the picture and the main variables exist in the detection can be used to distinguish the boundary of the surrounding color. Once, the converted grayscale image has been blurred, which can reduce the amount of calculation and facilitate subsequent detection and positioning. The specified method is applied to obtain the change amount of each pixel gradient, and the data conversion processing is running the condition of the ISSN: 2582-2012 (online) 
Journal of Artificial Intelligence and Capsule Networks (2020)

Vol.02/ No. 02

Pages: 77-99

http://irojournals.com/aicn/

DOI: https://doi.org/10.36548/jaicn.2020.2.002

variation of the results pixel gradient. The procedures mentioned previously can be established by the built-in edge detection libraries of OpenCV's. The machine vision system finally returns the results of numerical operation which are applied to draw the frame, successfully judge the square features, and completion to the judgement of object shape eventually.

\section{Implementation of Experiments and Results Discussion}

The methodologies for applying a 6-axis mechanical arm combine with computer vision on the object recognition in plane inspection are claimed and interpreted previously. Consequently, the experiments for validating the outcome with the hypothesis executed by the algorithm aforementioned is going to be described and implemented in this section.

\subsection{Assessment of the Implementation to Experiments}

There are five parts included in the structure of the 6-axis mechanical robot arm proposed in the article, including the control system, servo motor, mechanical structure, inductive element, and line control. Each part of the structure is described as follows, and the full diagram is shown in Fig. 10.

\subsubsection{Main Control System}

The key component that determines the driving function and command signal to control the operation of the robotic arm is the main control system. An excellent controller should have sufficient computing and data storage capacity plus the input and output interfaces. Hence, it

ISSN: 2582-2012 (online) 
Journal of Artificial Intelligence and Capsule Networks (2020)

Vol.02/ No. 02

Pages: 77-99

http://irojournals.com/aicn/

DOI: https://doi.org/10.36548/jaicn.2020.2.002

is able to implement the assigned functions with software programming. Traditionally, both the microcontrollers and DSP chips may not be able to load the performance and resources required for the overall design. As the utilization requirements, a most suitable controller according to the application fields is necessary. The control modular of Raspberry Pi-3 is chosen as the application tool in the article, and the assignment of GPIO pin for the inspection functions has been shown in Fig. 5 already.

\subsubsection{Servo motor}

The completion to the posture placement and movement trajectories for the robot arm is a servo motor. The achievement of the complex operations is including gripping, moving, and must cooperate through multiple motors. The stability of the servo motor is much higher than that of the stepper motor, since it can recognize the current motion status through the control loop.

\subsubsection{Mechanical structure}

Actually, the mechanical structure of the arm is copied by a 3D printer follows up the concept designed by the authors. It has a humanoid appearance but there are also irregular shapes and special designs for emphasizing functionality. As shown in Fig. 9, specified to claim that the structure of a likely human upper arm from 3D printer copying can be easily divided into shoulder, shoulder joint, elbow joint, wrist joint and finger.

ISSN: 2582-2012 (online) 
Journal of Artificial Intelligence and Capsule Networks (2020)

Vol.02/ No. 02

Pages: 77-99

http://irojournals.com/aicn/

DOI: https://doi.org/10.36548/jaicn.2020.2.002

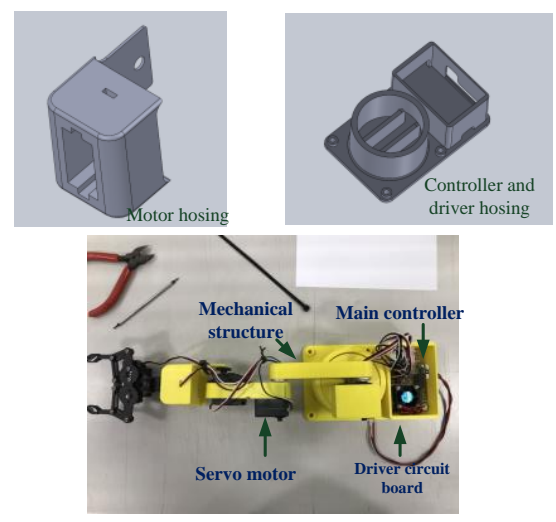

Fig. 9 The structure and parts of a likely human upper arm from 3D printer copying

In fact, corresponding to the rotating base, boom, arm and gripper are according to the needs of the task to perform appropriate structural modification and planning. The strictly requirement is matching the physical limitations of the motor component parameters and the scope of application. For considering the safety to protect the robot arm and the surroundings matters to avoid erroneous activity during the operation of the mechanism is definitely necessary.

\subsubsection{Inductive Components}

Study on the whole system is relying solely on motors and controllers for operation, its efficiency is much lower than that of man-machine cooperation systems equipped with inductive components. It needs to think about how to strengthen the external sensory behavior of the robotic arm, moreover, the data read from each kind of sensor by the control system to judge the environmental changes in the current movement status. So far, the robot arm is a deepened one while to feed back to all joints and perform relative strain behavior. This article, therefore, by using a web-camera that does not affect computing performance and it sets the ISSN: 2582-2012 (online) 
Journal of Artificial Intelligence and Capsule Networks (2020)

Vol.02/ No. 02

Pages: 77-99

http://irojournals.com/aicn/

DOI: https://doi.org/10.36548/jaicn.2020.2.002

resolution as 480 pixels.

\subsubsection{Line Control}

For comparison reason, the line control methods in the experiment is implemented with three types of motors, namely 'DC motors', 'stepper motors', and 'servo motors', respectively. The voltage is the most factor to control the speed of a DC motor. The current direction is applied to control the direction of the motor. The voltage source and the ground terminal can complete the dual-polarity control. Furthermore, the combination of the stepping motor and the control drive board relies on the power signal to be converted into angular displacement. The arm rotates a fixed angle according to the set displacement for achieving the positioning of the operation while receiving a power signal. At the same time, the frequency of the supplied power source can be controlled, if one wants to change the acceleration of rotation due to achieve the adjustment of the rotation speed. A four-phase five-wire control way can be worked in the condition of coil signal with pin 4 wire and the voltage source control 1 wire.

The rotation angle is controlled by the servo motor relies on the analysis of the pulse signal working cycle, and the 3-wire control can be completed by the voltage source as well as the ground terminal and the control pin. As the arrangement mentioned previous is mainly comprised with a 2-wire control DC motor, a stepping motor 5-wire control, and a 3-wire control servo motor. In order to save the input and output pin's number and make it is easily controlled. The method of selection the servo motor as the hardware structure and arranged the number of GPIO pins on the Raspberry Pi is very important.

\subsection{Results Discussion}

ISSN: 2582-2012 (online) 
Journal of Artificial Intelligence and Capsule Networks (2020)

Vol.02/ No. 02

Pages: 77-99

http://irojournals.com/aicn/

DOI: https://doi.org/10.36548/jaicn.2020.2.002

Based on the algorithms developed in the previous sections, the color characteristics of the test required considered first. Then, to define three basic colors of blue, red, green. There is as assessment build up three different directions of shade for the experiment.

Thereafter, the actual position is converted in the software to locate the target and the position of the object determined according to the characteristics of the target object, respectively. In this experiment, the blue square wood is used for testing, the coordinates of the four vertices and the center point of the rectangle are detected. It is known that follows up the algorithm the spatial position and the edge detection calculation method is adjusted, and the border is drawn after returning the numerical result. The correct position information and judgment feature result in the plane space can be obtained when the target object has noted. The image information accompanies with instructions after finishing the picture captured can be sent to the control system for operating. The specific method is to construct a group of 6-degree-of-freedom robotic arm with a USB interface. The webcam, which combined with the input control module and output control module of the embedded device, compiles and controls the mechanical arm in the programming language to correct the action according to the image information, after the target object is detected and positioned. It grabs and classifies the object to the specified area placement.

EXAMPLEP: "dark blue \# 0000FF”

By assuming the conditions including three different conditions of centering, right, and left, the position of the light source considers placed with the directions of forward light, left light, and right light depends on the projection angle. The results from different tests are composed to carry out 1260 times after the objects are placed in different positions described previously. Accordingly, the results of the arm grabs and subsequent statistics are shown in Fig. 10.

ISSN: 2582-2012 (online) 
Journal of Artificial Intelligence and Capsule Networks (2020)

Vol.02/ No. 02

Pages: 77-99

http://irojournals.com/aicn/

DOI: https://doi.org/10.36548/jaicn.2020.2.002

The experimental results of considering the conditions, which the object is located at the left and the light source is from the right side, show that the number of successes reaches 62 times. The object grabbed successfully are 61 times and the other 64 times while the light source is focused from left side and forward direction, respectively. The total number of the execution for Exp. "dark blue \# 0000FF" with different three light sources directions is 210 times. Thus, the number of successful captures is 187 times which is with an accuracy rate of $89 \%$. Comparing to the other case assuming that the target is located at the center position. The results from the experiment of "dark blue \# 0000FF" indicate the number of successes reaches 50 times, 60 times, and 58 times that the light source is considered from right, left sides, and the forward direction, respectively. At that time, the total number of such tests for the first three light sources was 210 times and the number of successful captures is 168 times. An accuracy rate of $80 \%$ is obtained as shown in Fig. 10. It is easily calculated from the results shown in Fig. 10 that the accuracy rate is with $75.2 \%$.

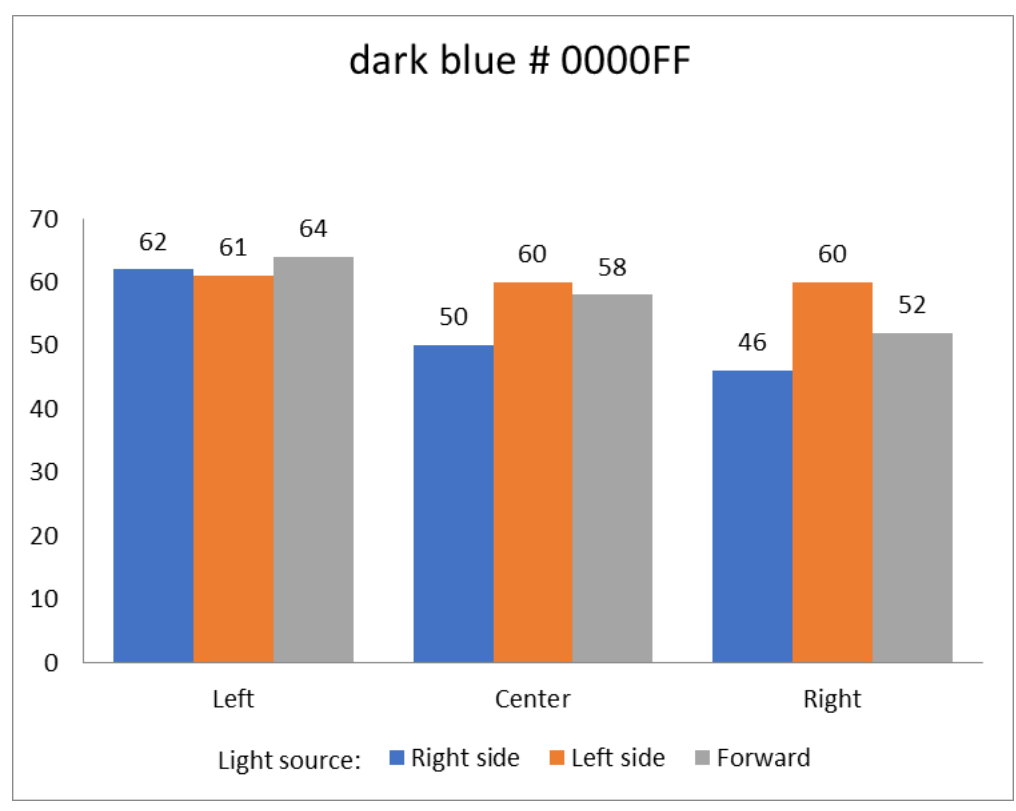

96

ISSN: 2582-2012 (online) 
Journal of Artificial Intelligence and Capsule Networks (2020)

Vol.02/ No. 02

Pages: 77-99

http://irojournals.com/aicn/

DOI: https://doi.org/10.36548/jaicn.2020.2.002

Fig. 10. The results of Exp. “dark blue \# 0000FF” with different light directions

\section{Conclusion}

The design to an intelligent vehicle system with the integration of electromechanical for planning to apply it to the operations in various fields is presented in the paper. Moreover, the main purpose of this work tries to avoid the complicated process with traditional manual adjustment or training. It is expected to achieve the purpose that the robotic arm can grab the target automatically, classify the target and place it in the specified area, and even accurately realize the classification through training to distinguish the characteristics of the target. Eventually, the mechanical arm's movement behavior is able to be corrected through a real-time image data feedback control system. The evaluation of performance for the purposed robot arm is held under some assumptions, and eventually it approves that the proposed algorithm has well known that the accuracy of target tracking definitely depends on both of two parameters include "object locations" and the "illustration direction" of light source. The difference will far from $75.2 \%$ to $89.0 \%$.

\section{References}

[1] Luis Variz, Luis Piardi, Pedro João Rodrigues, Paulo Leitão, "Machine Learning Applied to an Intelligent and Adaptive Robotic Inspection Station”, 2019 IEEE 17th International Conference on Industrial Informatics (INDIN), Vol. 1, 2019.

[2] Alexander Wong, Zhong Qiu Lin, Brendan Chwyl, "AttoNets: Compact and Efficient Deep Neural Networks for the Edge via Human-Machine Collaborative Design," 2019 IEEE/CVF Conference on Computer Vision and Pattern Recognition Workshops (CVPRW), 2019.

ISSN: 2582-2012 (online) 
Journal of Artificial Intelligence and Capsule Networks (2020)

Vol.02/ No. 02

Pages: 77-99

http://irojournals.com/aicn/

DOI: https://doi.org/10.36548/jaicn.2020.2.002

[3] Prachi P. Sonawane, Gayatri M. Phade, 'Motion Control of Robotic Arm For Micro positioning In Industrial Application”, 2018 International Conference On Advances in Communication and Computing Technology (ICACCT), Ahmednagar, India. pp. 553-558, Feb 8-9, 2018.

[4] Michal Ma'uga, Lazy pod Makytou, Slovakia, "Control and Positioning of Robotic Arm on CNC Cutting Machines and Their Applications in Industry Real-time Control System", Proceedings of the 29th International Conference 2018 Cybernetics \& Informatics (K\&I), Jan. 31-Feb. 3, 2018.

[5] Jin-Siang Shaw, Vipul Dubey, "Design of Servo Actuated Robotic Gripper Using Force Control for Range of Objects", Proceedings of 2016 International Conference on Advanced Robotics and Intelligent Systems, Taipei, Taiwan, 31 Aug.-2 Sep. 2016.

[6] Roland Szabó, and Aurel Gontean, "Robotic Arm Movement Using Color Detection with FPGA Vision," 2014 IEEE 20th International Symposium for Design and Technology in Electronic Packaging (SIITME), pp.117-122, Bucharest, Romania, Oct. 2014.

[7] Camshifthttps://fr.wikipedia.org/wiki/Camshift

[8] Ould-dris Nouar, Ganoun Ali and Canals Raphaël, "Improved Object Tracking with Camshift Algorithm", 2006 IEEE International Conference on Acoustics Speech and Signal Processing Proceedings.

\section{Author's Biography}

Joy Iong-Zong Chen, professor is the Department of Electrical Engineering, at Da-Yeh university, Taiwan is very much interested in performing researches in the artificial intelligence, his other areas of interest includes Artificial Intelligence, Robotics, Deep Learning, Cognitive Science, Ad-hoc Networks, Artificial Neural Network, Evolutionary

ISSN: 2582-2012 (online) 
Journal of Artificial Intelligence and Capsule Networks (2020)

Vol.02/ No. 02

Pages: 77-99

http://irojournals.com/aicn/

DOI: https://doi.org/10.36548/jaicn.2020.2.002

Computing, Speech Recognition and Autonomous System.

Jen-Ting Chang, is a Research Scholar in the Department of Electrical Engineering, at Da-Yeh University, his area of research includes Complex and Intelligent Systems, Networks, Recommendation System, Human-Computer Interface, Knowledge Representation, Pattern Recognition etc.

ISSN: 2582-2012 (online) 